\title{
Performance of Microflex LT Biotyper and VITEK MS for Routine Identification of Yeasts
}

\author{
Jung-Hyun Byun, M.D., A-Reum Yu, M.S., Myung Sook Kim, Ph.D., and Kyungwon Lee 마, M.D. \\ Department of Laboratory Medicine and Research Institute of Bacterial Resistance, Severance Hospital, Yonsei University College of Medicine, Seoul, Korea
}

\section{Dear Editor,}

The epidemiology of yeast infections is rapidly evolving, leading to the emergence of uncommon yeasts [1]. Rapid identification, followed by appropriate antimicrobial therapy, is associated with lower mortality [2]. Conventional phenotypic methods cannot differentiate certain yeast species accurately [3]. Matrix-assisted laser desorption ionization-time of flight mass spectrometry (MALDI-TOF MS) has been introduced in clinical microbiology to facilitate rapid yeast identification [3]. MALDI-TOF MS for yeast identification requires special preparation, similar to that for Mycobacterium species and gram-positive bacteria [4]. We compared the yeast identification capabilities of two MALDI-TOF systems- the Microflex LT Biotyper (Bruker Daltonics, Leipzig, Germany) and the VITEK MS (bioMerieux, Marcy-l'Étoile, France) — with respect to different sample preparation methods.

We included 208 yeast isolates collected from clinical samples at Severance Hospital between 2012 and 2015: blood $(\mathrm{N}=169)$, catheter $(\mathrm{N}=19)$, urine $(\mathrm{N}=12)$, sputum $(\mathrm{N}=6)$, and pus $(N=2)$. Yeasts were identified at isolation by conventional phenotypic methods, including the VITEK 2 YST card (bioMerieux, Durham, NC, USA). For the Biotyper analysis, on-plate formic acid extraction and in-tube formic acid/acetonitrile extraction were performed as previously described [5]. For the VITEK MS, only on-plate formic acid extraction was performed be- cause the in-tube method is not recommended by the manufacturer. When the yeast identification results of the VITEK 2 YST card and the two MALDI-TOF systems were consistent, they were considered reference identification. However, when the commercial system failed to identify the species or in cases of discordant results between the two MALDI-TOF systems, internal transcribed spacer (ITS) region sequencing was performed. This study was approved by the Institutional Review Board of Severance Hospital (2017-2752-001).

The Biotyper identification results for the two sample preparation methods are shown in Table 1. With the on-plate method, $95.7 \%$ of the isolates were correctly identified at the species level. With the in-tube extraction method, all isolates were correctly identified at the species level, consistent with previous reports [5-8]. The difference could be attributed to the ineffective lysis of the encapsulated yeast by the incomplete on-plate extraction method [9].

The Biotyper provides a species log score. A score $\geq 2.0$ indicates excellent identification at the species level. However, the data demonstrated correct identification of isolates with cut-off scores $<2.0$ as well. We derived an optimal cut-off score of $\geq 1.7$ for the Biotyper, using a ROC curve. This cut-off score demonstrated a sensitivity of $100.0 \%$ (95\% confidence interval [Cl] 86.3-100.0\%) and a specificity of $99.5 \%$ (95\% Cl 98.1-99.9\%).
Received: November 2, 2017

Revision received: February 20, 2018

Accepted: April 26, 2018

\section{Corresponding author: Kyungwon Lee}

(10) https://orcid.org/0000-0003-3788-2134

Department of Laboratory Medicine Yonsei University College of Medicine

50-1 Yonsei-ro, Seodaemun-gu, Seoul 03722, Korea

Tel: +82-2-2228-2446, Fax: +82-2-313-0956

E-mail: leekcp@yuhs.ac

\section{(c) Korean Society for Laboratory Medicine}

This is an Open Access article distributed under the terms of the Creative Commons Attribution Non-Commercial License (http://creativecommons.org/licenses/by-nc/4.0) which permits unrestricted non-commercial use, distribution, and reproduction in any medium, provided the original work is properly cited. 
Table 1. Microflex LT Biotyper identification scores using the on-plate and in-tube formic acid extraction methods

\begin{tabular}{|c|c|c|c|c|c|c|c|c|c|c|}
\hline \multirow{3}{*}{ Reference ID* (N tested) } & \multicolumn{10}{|c|}{$\mathrm{N}(\%)$ of isolates with Biotyper score } \\
\hline & \multicolumn{6}{|c|}{ On-plate method } & \multicolumn{4}{|c|}{ In-tube extraction method } \\
\hline & $\geq 2.0$ & $1.9-<2.0$ & $1.8-<1.9$ & $1.7-<1.8$ & $<1.7$ & № ID & $\geq 2.0$ & $1.9-<2.0$ & $1.8-<1.9$ & $1.7-<1.8$ \\
\hline \multicolumn{11}{|l|}{ Candida spp. } \\
\hline Candida albicans (65) & $34(52.3)$ & $25(38.5)$ & $3(4.6)$ & $3(4.6)$ & & & $65(100)$ & & & \\
\hline Candida tropicalis (38) & $8(21.1)$ & $15(39.5)$ & $11(28.9)$ & $1(2.6)$ & & $3(7.9)$ & $35(92.1)$ & $3(7.9)$ & & \\
\hline Candida glabrata (37) & $28(75.7)$ & $4(10.8)$ & $3(8.1)$ & $1(2.7)$ & $1(2.7)$ & & $37(100)$ & & & \\
\hline Candida parapsilosis (29) & $4(13.8)$ & $9(31)$ & $10(34.5)$ & $4(13.8)$ & & $2(6.9)$ & $15(51.7)$ & $8(27.6)$ & $4(13.8)$ & $2(6.9)$ \\
\hline Candida krusei (9) & $7(77.8)$ & $1(11.1)$ & & & & $1(11.1)$ & $7(77.8)$ & 2 (22.2) & & \\
\hline Candida Iusitaniae (7) & $4(57.1)$ & $2(28.6)$ & $1(14.3)$ & & & & $6(85.7)$ & $1(14.3)$ & & \\
\hline Candida guilliermondii (5) & $3(60)$ & $2(40)$ & & & & & $4(80)$ & $1(20)$ & & \\
\hline Candida dubliniensis (3) & $1(33.3)$ & $2(66.7)$ & & & & & $2(66.7)$ & $1(33.3)$ & & \\
\hline Candida kefyr (2) & $2(100)$ & & & & & & $2(100)$ & & & \\
\hline \multicolumn{11}{|l|}{ Non-Candida spp. } \\
\hline Cryptococcus neoformans (6) & $3(50)$ & & $1(16.7)$ & & & $2(33.3)$ & $6(100)$ & & & \\
\hline Trichosporon asahii (4) & $3(75)$ & & & & & $1(25)$ & $4(100)$ & & & \\
\hline Cryptococcus gattii (1) & $1(100)$ & & & & & & $1(100)$ & & & \\
\hline Cyberlindnera fabianii (1) & $1(100)$ & & & & & & $1(100)$ & & & \\
\hline Saccharomyces cerevisiae (1) & $1(100)$ & & & & & & $1(100)$ & & & \\
\hline Total (208) & $100(48.1)$ & $60(28.8)$ & $29(13.9)$ & $9(4.3)$ & $1(0.5)$ & $9(4.3)$ & $186(89.4)$ & $16(7.7)$ & $4(1.9)$ & $2(1)$ \\
\hline Cumulative Total & $100(48.1)$ & $160(76.9)$ & $189(90.8)$ & 198 (95.2) & $199(95.7)$ & $208(100)$ & $186(89.4)$ & 202 (97.1) & $206(99.0)$ & $208(100)$ \\
\hline
\end{tabular}

*If the identifications of the three methods were consistent, the result was considered a reference identification. When any of the results varied, ITS region sequencing was performed.

Abbreviation: ID, identification.

Table 2. Identification of clinical yeast isolates using the Microflex LT Biotyper, VITEK MS, and VITEK 2

\begin{tabular}{|c|c|c|c|c|c|c|}
\hline \multirow[b]{2}{*}{ Reference ID (N, ITS-tested N) } & \multicolumn{2}{|c|}{ Microflex LT Biotyper } & \multicolumn{2}{|c|}{ VITEK MS } & \multicolumn{2}{|c|}{ VITEK 2} \\
\hline & $\begin{array}{l}\text { Correct IDs at the } \\
\text { species level }\end{array}$ & Discordant IDs & $\begin{array}{l}\text { Correct IDs at the } \\
\text { species level }\end{array}$ & Discordant IDs & $\begin{array}{l}\text { Correct IDs at the } \\
\text { species level }\end{array}$ & Discordant IDs \\
\hline Candida albicans $(65,1)$ & $65(100)$ & & $65(100)$ & & $64(98.5)$ & $1(1.5)$ \\
\hline Candida tropicalis $(38,1)$ & $37(100)$ & & $37(100)$ & & $37(97.4)$ & $1(2.6)$ \\
\hline Candida glabrata $(37,0)$ & $37(100)$ & & $37(100)$ & & $37(100)$ & \\
\hline Candida parapsilosis $(29,3)$ & $29(100)$ & & $29(100)$ & & $24(82.8)$ & $5(17.2)$ \\
\hline Candida krusei $(9,1)$ & $9(100)$ & & $9(100)$ & & $7(77.8)$ & $2(22.2)$ \\
\hline Candida Iusitaniae $(7,2)$ & $7(100)$ & & $7(100)$ & & $5(71.4)$ & $2(28.6)$ \\
\hline Candida guilliermondii $(5,3)$ & $5(100)$ & & $5(100)$ & & $1(20.0)$ & $4(80.0)$ \\
\hline Candida dubliniensis $(3,1)$ & $3(100)$ & & $3(100)$ & & $2(66.7)$ & $1(33.3)$ \\
\hline Candida kefyr $(2,0)$ & $2(100)$ & & $2(100)$ & & $2(100)$ & \\
\hline Cryptococcus neoformans $(6,1)$ & $6(100)$ & & $6(100)$ & & $5(83.3)$ & $1(16.7)$ \\
\hline Trichosporon asahii $(4,1)$ & $4(100)$ & & $4(100)$ & & $3(75.0)$ & $1(25.0)$ \\
\hline Cryptococcus gattii $(1,1)$ & $1(100)$ & & $0(0)$ & $1(100)$ & & $1(100)$ \\
\hline Cyberlindnera fabianii $(1,1)$ & $1(100)$ & & $1(100)$ & & & $1(100)$ \\
\hline Saccharomyces cerevisiae $(1,0)$ & $1(100)$ & & $1(100)$ & & $1(100)$ & \\
\hline Total $(208,20)$ & $208(100)$ & & $207(99.5)$ & $1(0.5)$ & $188(90.4)$ & $20(9.6)$ \\
\hline
\end{tabular}

Values are presented as N (\%).

Abbreviations: ID, identification; ITS, internal transcribed spacer; MS, mass spectrometry. 
When this cut-off was applied, 94.7\% of the isolates were correctly identified at the species level using the Biotyper system with the on-plate method. This rate increased to $100 \%$ using the same system with the in-tube method. With this cut-off, the yeast identification ability of the Biotyper was comparable with that of VITEK MS. The final identification rates were $100.0 \%$ and $99.5 \%$ for the Biotyper and VITEK MS, respectively (Table 2). VITEK MS provided correct identification at the species level for all 208 isolates, except Cryptococcus gattii, which is not included in the VITEK MS database. The correct identification rate of the VITEK 2 system with the YST card was $90.4 \%$.

Previous studies have suggested various cut-off values $<2$ [3, 10], and we found that the laboratory-validated cut-off value yielded a higher identification rate without compromising accuracy. Lee et al [5] reported correct identification rates of $91.4 \%$ and $97.8 \%$ using the Biotyper ( $\geq 1.7$ ) and the VITEK MS, respectively, with the on-plate method. Their results included 37 uncommon yeast species, which might explain why their correct identification rates were slightly lower than ours (94.7\% and 99.5\%).

The on-plate method is preferred to in-tube extraction method. The latter method is time-consuming and laborious, although, traditionally, it has provided better identification results in the clinical laboratory. Lower cut-off scores using the on-plate method have resulted in greater consistency between the results of the two methods, except for $C$. neoformans. Moreover, the on-plate method may reduce the time and labor required to perform retests that are often required with the in-tube method or other complementary tests, such as ITS region sequencing.

In summary, the Biotyper and VITEK MS platforms demonstrated comparable performance for routine identification of clinically common yeasts ( $100 \%$ vs $99.5 \%$, respectively). VITEK MS yields accurate results using the simple on-plate method. The Biotyper requires the in-tube extraction method to reach a score $\geq 2.0$; however, with the application of a flexible cut-off value ( $\geq 1.7$ ), the on-plate method is sufficient to achieve a correct identification rate of $>95 \%$.

\section{Authors' Disclosure of Potential Conflict of Interest}

No potential conflicts of interest relevant to this article were reported.

\section{REFERENCES}

1. Miceli MH, Díaz JA, Lee SA. Emerging opportunistic yeast infections. Lancet Infect Dis 2011;11:142-51.

2. Morrell M, Fraser VJ, Kollef MH. Delaying the empiric treatment of Candida bloodstream infection until positive blood culture results are obtained: a potential risk factor for hospital mortality. Antimicrob Agents Chemother 2005;49:3640-5.

3. Dhiman N, Hall L, Wohlfiel SL, Buckwalter SP, Wengenack NL. Performance and cost analysis of matrix-assisted laser desorption ionizationtime of flight mass spectrometry for routine identification of yeast. J Clin Microbiol 2011;49:1614-6.

4. Theel ES, Schmitt BH, Hall L, Cunningham SA, Walchak RC, Patel R, et al. Formic acid-based direct, on-plate testing of yeast and Corynebacterium species by Bruker Biotyper matrix-assisted laser desorption ionization-time of flight mass spectrometry. J Clin Microbiol 2012;50:3093-5.

5. Lee HS, Shin JH, Choi MJ, Won EJ, Kee SJ, Kim SH, et al. Comparison of the Bruker Biotyper and VITEK MS matrix-assisted laser desorption/ ionization time-of-flight mass spectrometry systems using a formic acid extraction method to identify common and uncommon yeast isolates. Ann Lab Med 2017;37:223-30.

6. Chao QT, Lee TF, Teng SH, Peng LY, Chen PH, Teng LJ, et al. Comparison of the accuracy of two conventional phenotypic methods and two MALDITOF MS systems with that of DNA sequencing analysis for correctly identifying clinically encountered yeasts. PLoS One 2014;9:e109376.

7. McTaggart LR, Lei E, Richardson SE, Hoang L, Fothergill A, Zhang SX. Rapid identification of Cryptococcus neoformans and Cryptococcus gattii by matrix-assisted laser desorption ionization-time-of-flight mass spectrometry. J Clin Microbiol 2011;49:3050-3.

8. Pence MA, McElvania TeKippe E, Wallace MA, Burnham CA. Comparison and optimization of two MALDI-TOF MS platforms for the identification of medically relevant yeast species. Eur J Clin Microbiol Infect Dis 2014;33:1703-12.

9. Cassagne C, Cella AL, Suchon P, Normand AC, Ranque S, Piarroux R. Evaluation of four pretreatment procedures for MALDI-TOF MS yeast identification in the routine clinical laboratory. Med Mycol 2013;51:371-7.

10. Vlek A, Kolecka A, Khayhan K, Theelen B, Groenewald M, Boel E, et al. Interlaboratory comparison of sample preparation methods, database expansions, and cutoff values for identification of yeasts by matrix-assisted laser desorption ionization-time of flight mass spectrometry using a yeast test panel. J Clin Microbiol 2014;52:3023-9. 